\title{
Anxiety sensitivity as a predictor of broad dimensions of psychopathology after cognitive behavioral therapy for panic disorder
}

This article was published in the following Dove Press journal:

Neuropsychiatric Disease and Treatment

13 July 2017

Number of times this article has been viewed

\author{
Keiko Ino' \\ Sei Ogawa' \\ Masaki Kondo' \\ Risa Imai' \\ Toshitaka li' \\ Toshi A Furukawa ${ }^{2}$ \\ Tatsuo Akechi' \\ 'Department of Psychiatry and \\ Cognitive-Behavioral Medicine, \\ Nagoya City University Graduate \\ School of Medical Sciences, Nagoya, \\ 2Department of Health Promotion \\ and Human Behavior, Kyoto University \\ Graduate School of Medicine/School \\ of Public Health, Kyoto, Japan
}

Background: Panic disorder (PD) is a common disease and presents with broad dimensions of psychopathology. Cognitive behavioral therapy (CBT) is known to improve these broad dimensions of psychopathology in addition to PD symptoms. However, little is known about the predictors of treatment response in comorbid psychiatric symptoms after CBT for PD. Recent studies suggest that anxiety sensitivity (AS) may be a key vulnerability for PD. This study aimed to examine AS as a predictor of broad dimensions of psychopathology after CBT for PD.

Materials and methods: In total, 118 patients with PD were treated with manualized group CBT. We used multiple regression analysis to examine the associations between 3 Anxiety Sensitivity Index (ASI) factors (physical concerns, mental incapacitation concerns, and social concerns) at baseline and the subscales of the Symptom Checklist-90 Revised (SCL-90-R) at endpoint.

Results: Low levels of social concerns at baseline predicted low levels on 5 SCL-90-R subscales after CBT: interpersonal sensitivity, depression, hostility, paranoid ideation, and psychosis. High levels of mental incapacitation concerns significantly predicted low levels on 3 SCL-90-R subscales after treatment: interpersonal sensitivity, hostility, and paranoid ideation. Physical concerns at baseline did not predict broad dimensions of psychopathology.

Conclusion: This study suggested that the social concerns and mental incapacitation concerns subscales of the ASI at baseline predicted several dimensions of psychopathology after CBT for PD. To improve comorbid psychopathology, it may be useful to direct more attention to these ASI subscales.

Keywords: panic disorder, anxiety sensitivity, comorbid psychopathology, predictor, cognitive behavioral therapy

\section{Introduction}

Panic disorder (PD) is a common psychiatric problem with a lifetime prevalence of approximately $3 \% .{ }^{1}$ Fifty to eighty percent of PD patients meet criteria for other diagnoses, chiefly other anxiety or mood disorders. ${ }^{2,3}$ PD with comorbid psychiatric symptoms is considered to be a severe condition. For example, comorbidity between PD and depressive disorder is thought to be associated with symptom severity and poorer outcomes. ${ }^{4} \mathrm{PD}$ also presents subthreshold psychiatric symptoms such as somatization, phobic anxiety, interpersonal anxiety, depression, and so on.

The efficacy of cognitive behavioral therapy (CBT) has been demonstrated in PD. ${ }^{5}$ Several studies have shown that CBT targeted for an anxiety disorder produced positive benefits on broad dimensions of psychopathology. ${ }^{2,5,6}$ To provide more effective treatment for broad dimensions of psychopathology, it is useful to examine baseline predictors of this psychopathology after CBT for PD. However, no specific predictor
Department of Psychiatry and CognitiveBehavioral Medicine, Nagoya City University Graduate School of Medical Sciences, Mizuho-cho, Mizuho-ku, Nagoya, Japan

Tel +8 I 52853827 I

Fax +8I 528520837

Email miniture_flute@hotmail.com 
for broad dimensions of psychopathology after CBT for PD has yet been found.

Patients with PD with psychiatric comorbidity have been found to have elevated levels of anxiety sensitivity (AS). ${ }^{6}$ AS refers to a fear of anxiety-related sensations. ${ }^{7}$ Higher levels of AS are observed in individuals with anxiety disorders compared with healthy individuals. ${ }^{8}$ AS is also thought to be a vulnerability factor in the pathogenesis of many psychiatric disorders. ${ }^{9}$ Moreover, high levels of AS are prospectively related to anxiety symptoms ${ }^{9}$ and depressive symptoms. ${ }^{10}$ Recent studies revealed each subscale of Anxiety Sensitivity Index (ASI) to be more perceptively related to panic course or severity than total score of ASI. ${ }^{11,12}$ Previous studies have reported the predictive value of AS in the occurrence of psychiatric symptoms. However, no study has examined whether or not AS predicted outcomes in some dimensions of psychopathology after CBT for PD.

This study aimed to determine if AS predicted improvement in residual broad dimensions of psychopathology after CBT for PD.

\section{Materials and methods}

\section{Participants}

Participants were recruited between September 2005 and May 2013, and a consecutive series of 132 patients entered the CBT program. Eligible participants were 132 patients with PD who attended a group CBT program. All the recruited patients met specific inclusion criteria: 1) principal Axis I diagnosis of PD, with or without agoraphobia, according to the Diagnostic and Statistical Manual of Mental Disorders, Fourth Edition (DSM-IV) criteria, as assessed by the Structured Clinical Interview for DSM-IV (SCID); ${ }^{13}$ 2) highly motivated to receive CBT; and 3) free from benzodiazepine use before CBT entry, as these drugs interact negatively with exposure treatments. ${ }^{14}$ Exclusion criteria were 1) history of psychosis and 2) current substance use disorder. Use of antidepressants was permitted throughout the CBT program, as these drugs do not interfere with CBT treatment. ${ }^{15}$ All participants provided written informed consent after receiving a full explanation of the study's purpose and procedures. The study protocol was approved by the Ethics Committee of Nagoya City University Graduate School of Medical Sciences.

\section{Procedure}

Treatment

The CBT program used in this study was based on the CBT treatment manual for PD, written by Andrews et al. ${ }^{16}$
The program was delivered to groups of 3-4 patients by 1 principal therapist and 1 co-therapist. The therapist was a psychiatrist or a clinical psychologist with more than 2 years of clinical experience. Fourteen therapists conducted CBT as a principal therapist; 8 therapists were male and 6 were female. Principal therapist should have finished CBT workshops and training period as a co-therapist. The treatment comprised 10 sessions, with each session scheduled to last 2 hours. The first 2 sessions included psychoeducation about the nature of anxiety, panic symptoms, and agoraphobia and provided a rationale for breathing retraining. Cognitive restructuring, in vivo exposure, and interoceptive exposure were introduced from the third session. The participants were asked to try to formulate rational thoughts and to conduct exposure tasks to reproduce external and interoceptive phobic cues during and between sessions. In addition, the participants were assigned homework after each session.

\section{Assessment}

All the participants underwent a semi-structured interview before the CBT program, including the anxiety and mood disorders section of the SCID and the Panic Disorder Severity Scale (PDSS). These interviews were conducted by one of the clinicians in charge of the program. At the start of the program, all the participants completed self-report questionnaires. To measure AS, the participants were asked to complete the ASI. Comorbid psychiatric symptoms were assessed with the Symptom Checklist-90 Revised (SCL90-R). The PDSS, ASI, and SCL-90-R were repeated at the end of the CBT program.

\section{Measures and rating scales ASI}

The ASI is widely used to evaluate AS. The ASI is a 16-item questionnaire, ${ }^{7}$ with each item rated on a 5-point scale from 0 (very little) to 4 (very much). Examples of items are: "It scares me when I feel 'shaky' (trembling)"; "It is important for me not to appear nervous"; and "When I cannot keep my mind on a task, I worry that I might be going crazy". Higher scores represent greater AS. In this study, we used the lower order factors, namely, physical concerns, mental incapacitation concerns, and social concerns. ${ }^{17}$ Physical concerns measure fears that physical reactions may lead to catastrophic outcomes. Mental incapacitation concerns capture fears about insanity or going crazy, and social concerns measure fears of having anxiety noticed by others. ${ }^{18}$ Adequate inter-rater reliability and validity have been reported for both the original ${ }^{19}$ and the Japanese versions of the ASI. ${ }^{20}$ 


\section{PDSS}

The PDSS is an interview-based, 7-item scale used to assess PD severity. ${ }^{21}$ The interviewer (clinician) rates the severity of $7 \mathrm{PD}$ features on a scale ranging from 0 (none) to 4 (extreme). The 7 areas covered are frequency of panic attacks, distress during panic attacks, anticipatory anxiety, agoraphobic fear/ avoidance, interoceptive fear/avoidance, work impairment/ distress, and impairment of social functioning. Adequate inter-rater reliability and validity have been reported for both the original ${ }^{21}$ and the Japanese versions of the PDSS. ${ }^{22}$

\section{SCL-90-R}

The SCL-90-R is commonly used to assess general psychopathology. ${ }^{23}$ It contains 90 items divided into 9 subscales: somatization, obsessive-compulsive, interpersonal sensitivity, depression, anxiety, hostility, phobic anxiety, paranoid ideation, and psychosis. The summary score, the Global Severity Index (GSI), combines information on the number of symptoms and intensity of perceived distress. Each item is scored from 0 (not at all) to 5 (extremely), with the average of relevant items taken to be the subscale score. The reliability and validity of the Japanese version of the SCL-90-R have been reported. ${ }^{24}$

\section{Statistical analysis}

We used Statistical Package for the Social Sciences (SPSS) Version 21.0 (IBM SPSS Statistics for Mac; IBM Corp., Armonk, NY, USA) for the statistical analyses. ${ }^{25}$ First, we used $\chi^{2}$ tests and analysis of variance to compare the demographic and clinical data between participants who completed the program and those who did not. Second, we used paired Student's $t$-tests to compare the baseline and endpoint symptoms scores for those who completed the program. Third, to examine the predictors of the indices of broad dimensions of psychopathology, we performed multiple linear regression analyses using age, sex, total PDSS score at baseline, and 3 ASI factors (physical concerns, mental incapacitation concerns, and social concerns) as independent variables, and the subscales of SCL-90-R at endpoint as dependent variables, while controlling for SCL-90-R baseline subscale scores. All statistical tests were 2-tailed, and the level of statistical significance was set at $P=0.05$ (uncorrected, 2-sided).

\section{Results}

Seven $(5.3 \%)$ of the 132 patients who started the CBT program dropped out prematurely. A further 7 patients had missing data, resulting in 118 patients being included in the present analysis. Table 1 shows participants' demographic and clinical characteristics. Twenty-one patients (15.9\%) had comorbidities; 9 of specific phobia, 4 of major depression, 2 of dysthymic disorder, 3 of general anxiety disorder, 1 posttraumatic stress disorder, and 1 bipolar disorder. Fiftyfour patients $(40.9 \%)$ had received medication when CBT started, while 51 patients had received antidepressants and 10 patients received sleeping agents.

Table 2 presents participants' broad dimensions of psychopathology. No statistically significant differences were seen among the subgroups.

Table 3 shows the pretreatment and posttreatment symptom scale scores. All the posttreatment scores were significantly lower than the pretreatment scores $(P<0.01)$.

Table 4 shows the predictors of broad dimensions of psychopathology. Low levels of social concerns at baseline predicted low levels on 5 SCL-90-R subscales after CBT: interpersonal sensitivity, depression, hostility, paranoid ideation, and GSI. Moreover, high levels of mental incapacitation concerns significantly predicted low levels on $3 \mathrm{SCL}-$ 90-R subscales after treatment: interpersonal sensitivity, hostility, and paranoid ideation. Physical concerns at baseline did not predict any dimensions of psychopathology.

Table I Participants' baseline characteristics $(\mathrm{N}=132)$

\begin{tabular}{|c|c|c|c|c|}
\hline & $\begin{array}{l}\text { Completed CBT } \\
(\mathbf{N}=|| 8)\end{array}$ & $\begin{array}{l}\text { Completed CBT with } \\
\text { missing data }(\mathbf{N}=7)\end{array}$ & $\begin{array}{l}\text { Dropped out } \\
(\mathbf{N}=7)\end{array}$ & P-value \\
\hline Female, N (\%) & $88(75.6)$ & $6(85.7)$ & $4(57.1)$ & 0.47 \\
\hline Mean age, years (SD) & $36.8(11.4)$ & $37.7(14.9)$ & $33.0(11.9)$ & 0.68 \\
\hline Duration of the disease, years (SD) & $7.3(6.3)$ & $5.3(3.3)$ & $4.9(5.2)$ & 0.44 \\
\hline Agoraphobia (\%) & III (94.0) & $6(85.7)$ & $7(100)$ & 0.53 \\
\hline PDSS (SD) & $12.97(4.89)$ & $|4.7|(5.16)$ & $13.86(6.28)$ & 0.62 \\
\hline ASI total (SD) & $28.03(14.02)$ & $21.43(13.75)$ & $17.00(14.17)$ & 0.07 \\
\hline ASI physical concerns (SD) & I $6.67(7.70)$ & $14.43(10.13)$ & $10.71(7.11)$ & 0.12 \\
\hline ASI mental incapacitation concerns (SD) & $4.96(4.47)$ & $3.14(3.19)$ & $2.14(4.81)$ & 0.17 \\
\hline ASI social concerns (SD) & $6.31(4.15)$ & $3.86(3.13)$ & $4.14(3.63)$ & 0.14 \\
\hline
\end{tabular}

Abbreviations: CBT, cognitive behavioral therapy; SD, standard deviation; PDSS, Panic Disorder Severity Scale; ASI, Anxiety Sensitivity Index. 
Table 2 Baseline SCL-90- $\mathrm{R}$ subscale scores $(\mathrm{N}=132)$

\begin{tabular}{|c|c|c|c|c|}
\hline & $\begin{array}{l}\text { Completed CBT } \\
(\mathrm{N}=\mid 18)\end{array}$ & $\begin{array}{l}\text { Completed CBT with } \\
\text { missing data }(\mathrm{N}=7)\end{array}$ & $\begin{array}{l}\text { Dropped out } \\
(N=7)\end{array}$ & $P$-value \\
\hline SCL-90-R somatization (SD) & $1.17(0.83)$ & $0.82(0.80)$ & $\mathrm{I} .46(0.8 \mathrm{I})$ & 0.35 \\
\hline SCL-90-R obsessive-compulsive (SD) & $1.20(0.82)$ & $0.76(1.07)$ & $0.80(0.53)$ & 0.20 \\
\hline SCL-90-R interpersonal sensitivity (SD) & $1.09(0.79)$ & $0.71(0.89)$ & $1.10(0.90)$ & 0.49 \\
\hline SCL-90-R depression (SD) & $1.18(0.82)$ & $0.60(0.59)$ & $1.26(0.88)$ & 0.18 \\
\hline SCL-90-R anxiety (SD) & $1.23(0.86)$ & $0.91(0.77)$ & I.II (0.94) & 0.60 \\
\hline SCL-90-R hostility (SD) & $0.7 \mathrm{I}(0.66)$ & $0.43(0.59)$ & $0.74(0.92)$ & 0.55 \\
\hline SCL-90-R phobic anxiety (SD) & $\mathrm{I} .40(0.95)$ & $1.69(0.90)$ & $1.57(1.05)$ & 0.67 \\
\hline SCL-90-R paranoid ideation (SD) & $0.64(0.69)$ & $0.50(0.84)$ & $0.40(0.48)$ & 0.62 \\
\hline SCL-90-R psychosis (SD) & $0.60(0.56)$ & $0.37(0.60)$ & $0.47(0.53)$ & 0.51 \\
\hline SCL-90-R GSI (SD) & $1.06(0.67)$ & $0.76(0.7 I)$ & $1.05(0.70)$ & 0.52 \\
\hline
\end{tabular}

Abbreviations: SCL-90-R, Symptom Checklist-90 Revised; CBT, cognitive behavioral therapy; SD, standard deviation; GSI, Global Severity Index.

High PDSS total scores predicted high levels of 6 SCL90-R subscales: obsessive-compulsive, depression, anxiety, phobic anxiety, psychosis, and GSI.

\section{Discussion}

The present study demonstrated that the social concerns and mental incapacitation concerns subscales of the ASI at baseline predicted several dimensions of psychopathology after CBT for PD. However, the physical concerns subscale of the ASI at baseline did not predict any dimensions of psychopathology. These results were consistent with previous findings that ASI-physical scores were strongly associated with PD symptoms, whereas ASI-cognitive scores were associated with generalized anxiety disorder symptoms and major depression symptoms, and ASI-social scores were associated with social phobia symptoms. ${ }^{26}$

Our results supported the suggestion that AS may be an important predictor of the clinical course of PD. ${ }^{11}$ Our finding that low levels of social concerns at baseline predicted high levels in comorbid psychopathology after CBT suggested that people with high AS for social factors tend to have difficulty joining group work such as our CBT program or going out for in vivo exposure. Moreover, high levels of mental incapacitation concerns significantly predicted low levels on several SCL-90-R subscales. CBT for PD aims to change catastrophic cognitions, ${ }^{27}$ and a previous study demonstrated that reductions of ASI cognitive scores mediated successful treatment outcomes. ${ }^{28}$ Patients with PD who have high mental incapacitation concerns may therefore benefit from CBT. To improve comorbid psychopathology, it may be useful to pay more attention to 2 ASI factors: social concerns and mental incapacitation concerns.

\section{Limitations}

The present study has several limitations. First, the study did not include a control group, and analyses of the effects of treatment relied on within-group changes. Second, the rules regarding medication were not precise. Third, we lacked follow-up data and therefore could not determine the longterm effect on comorbid psychopathology. Fourth, this study was conducted between 2005 and 2013 using the DSM-IV.

Table 3 Pretreatment and posttreatment rating scale scores $(\mathrm{N}=\mathrm{I} \mid 8)$

\begin{tabular}{llll}
\hline & Pretreatment & Posttreatment & $\begin{array}{l}\text { Effect size } \\
\text { (95\% confidence interval) }\end{array}$ \\
\hline $\begin{array}{l}\text { PDSS (SD) } \\
\text { SCL-90-R }\end{array}$ & $12.97(4.89)$ & $6.04(3.83)$ & $-1.58(-1.87$ to -1.28$)$ \\
$\quad$ Somatization (SD) & $1.17(0.83)$ & $0.87(0.79)$ & $-0.37(-0.63$ to $-0.1 \mathrm{I})$ \\
Obsessive-compulsive (SD) & $1.20(0.82)$ & $0.93(0.78)$ & $-0.34(-0.59$ to -0.08$)$ \\
Interpersonal sensitivity (SD) & $1.09(0.79)$ & $0.80(0.70)$ & $-0.39(-0.65$ to -0.13$)$ \\
Depression (SD) & $1.18(0.82)$ & $0.86(0.75)$ & $-0.41(-0.67$ to -0.15$)$ \\
Anxiety (SD) & $1.23(0.86)$ & $0.90(0.8 \mathrm{I})$ & $-0.40(-0.65$ to -0.14$)$ \\
Hostility (SD) & $0.71(0.66)$ & $0.53(0.59)$ & $-0.29(-0.54$ to -0.03$)$ \\
Phobic anxiety (SD) & $1.40(0.95)$ & $0.83(0.83)$ & $-0.64(-0.90$ to -0.38$)$ \\
Paranoid ideation (SD) & $0.64(0.69)$ & $0.45(0.56)$ & $-0.30(-0.56$ to -0.04$)$ \\
Psychosis (SD) & $0.60(0.56)$ & $0.38(0.45)$ & $-0.43(-0.69$ to -0.17$)$ \\
GSI (SD) & $1.06(0.66)$ & $0.76(0.61)$ & $-0.47(-0.73$ to -0.21$)$ \\
\hline
\end{tabular}

Abbreviations: PDSS, Panic Disorder Severity Scale; SD, standard deviation; SCL-90-R, Symptom Checklist-90 Revised; GSI, Global Severity Index. 
Table 4 Predictors at baseline for Symptom Checklist-90 Revised subscale scores after CBT (N=I I8)

\begin{tabular}{lllllllllll}
\hline & Somatization & $\begin{array}{l}\text { Obsessive- } \\
\text { compulsive }\end{array}$ & Interpersonal & Depression & Anxiety & Hostility & $\begin{array}{l}\text { Phobic } \\
\text { anxiety }\end{array}$ & $\begin{array}{l}\text { Paranoid } \\
\text { ideation }\end{array}$ & $\begin{array}{l}\text { Psychosis } \\
\text { GSI }\end{array}$ \\
\hline Baseline & $0.64^{* *}$ & $0.52^{* *}$ & $0.43^{* *}$ & $0.47^{* *}$ & $0.33^{* *}$ & $0.48^{* *}$ & $0.55^{* *}$ & $0.53^{* *}$ & $0.53^{* *}$ & $0.49^{* *}$ \\
Sex & 0.02 & -0.00 & 0.08 & -0.02 & -0.00 & 0.08 & 0.04 & 0.06 & 0.03 & 0.02 \\
Age (years) & 0.05 & 0.04 & -0.05 & 0.09 & 0.03 & -0.01 & -0.02 & -0.06 & 0.01 & 0.00 \\
PDSS & 0.05 & $0.16^{*}$ & 0.08 & $0.17^{*}$ & $0.20^{*}$ & 0.13 & $0.20^{*}$ & 0.11 & $0.19^{* *}$ & $0.16^{*}$ \\
ASI-physical & 0.11 & 0.11 & 0.17 & 0.01 & 0.17 & 0.10 & 0.10 & 0.09 & 0.05 & 0.13 \\
ASI-mental & -0.05 & -0.09 & $-0.24^{*}$ & -0.11 & 0.16 & $-0.23^{*}$ & -0.11 & $-0.24^{*}$ & 0.01 & -0.09 \\
ASI-social & 0.08 & 0.12 & $0.32^{* *}$ & $0.28^{* *}$ & 0.05 & $0.25^{*}$ & 0.10 & $0.26^{* *}$ & 0.15 & $0.19^{*}$ \\
Adjusted $R^{2}$ & 0.50 & 0.43 & 0.46 & 0.40 & 0.45 & 0.31 & 0.47 & 0.45 & 0.51 & 0.49 \\
\hline
\end{tabular}

Notes: The table shows the standardized beta coefficients. $* P<0.05, * * P<0.01$.

Abbreviations: CBT, cognitive behavioral therapy; GSI, Global Severity Index; PDSS, Panic Disorder Severity Scale; ASI, Anxiety Sensitivity Index.

The Japanese version of the DSM-5 was published in 2014; therefore, we could not use DSM-5 criteria.

\section{Conclusion}

This study suggests that social concerns and mental incapacitation concerns at baseline may predict broad dimensions of psychopathology after CBT for PD. Therefore, it may be useful to direct more attention to these 2 ASI subscales to improve comorbid psychiatric symptoms.

\section{Acknowledgment}

This study was supported by a Grant-in-Aid from the Ministry of Education, Culture, Sports, Science and Technology, Japan (23530910).

\section{Author contributions}

This work was carried out in collaboration between all the authors. KI was the primary investigator for this study. TA initiated and supervised the overall research project. All the authors took part in the clinical investigation (diagnosis, treatment, and assessment). All authors contributed toward data analysis, drafting and revising the paper and agree to be accountable for all aspects of the work.

\section{Disclosure}

The authors report no conflicts of interest in this work.

\section{References}

1. Kessler RC, Chiu WT, Demler O, Merikangas KR, Walters EE. Prevalence, severity, and comorbidity of 12-month DSM-IV disorders in the National Comorbidity Survey Replication. Arch Gen Psychiatry. 2005;62: 617-627.

2. Tsao JC, Lewin MR, Craske MG. The effects of cognitive-behavior therapy for panic disorder on comorbid conditions. J Anxiety Disord. 1998; $12: 357-371$

3. Brown TA, Antony MM, Barlow DH. Diagnostic comorbidity in panic disorder: effect on treatment outcome and course of comorbid diagnoses following treatment. J Consult Clin Psychol. 1995;63:408-418.
4. Noyes R Jr, Reich J, Christiansen J, Suelzer M, Pfohl B, Coryell WA Outcome of panic disorder. Relationship to diagnostic subtypes and comorbidity. Arch Gen Psychiatry. 1990;47:809-818.

5. Craske MG, Farchione TJ, Allen LB, Barrios V, Stoyanova M, Rose R. Cognitive behavioral therapy for panic disorder and comorbidity: more of the same or less of more? Behav Res Ther. 2007;45:1095-1109.

6. Tsao JC, Mystkowski JL, Zucker BG, Craske MG. Effects of cognitivebehavioral therapy for panic disorder on comorbid conditions: replication and extension. Behav Ther. 2002;33(4):493-509.

7. Reiss S, Peterson RA, Gursky DM, McNally RJ. Anxiety sensitivity, anxiety frequency and the prediction of fearfulness. Behav Res Ther. 1986; 24:1-8.

8. Taylor S, Cox BJ. An expanded anxiety sensitivity index: evidence for a hierarchic structure in a clinical sample. J Anxiety Disord. 1998;12: $463-483$.

9. Schmidt NB, Mitchell MA, Richey JA. Anxiety sensitivity as an incremental predictor of later anxiety symptoms and syndromes. Compr Psychiatry. 2008;49:407-412.

10. Cox BJ, Fuentes K, Borger SC, Taylor S. Psychopathological correlates of anxiety sensitivity: evidence from clinical interviews and self-report measures. J Anxiety Disord. 2001;15:317-332.

11. Benítez CI, Shea MT, Raffa S, et al. Anxiety sensitivity as a predictor of the clinical course of panic disorder: a 1-year follow-up study. Depress Anxiety. 2009;26:335-342.

12. Osma López J, Barrada González JR, García-Palacios A, Botella Arbona C. Influence of vulnerability factors in panic disorder severity. Psicothema. 2016;28:167-173.

13. First MB. Structured Clinical Interview for DSM-IV Axis I Disorders: SCID - I: Clinician Version: Administration Booklet. Washington, DC: American Psychiatric Press; 1997.

14. Watanabe N, Churchill R, Furukawa TA. Combined psychotherapy plus benzodiazepines for panic disorder. Cochrane Database Syst Rev. 2009:CD005335.

15. Furukawa TA, Watanabe N, Churchill R. Psychotherapy plus antidepressant for panic disorder with or without agoraphobia: systematic review. Br J Psychiatry. 2006;188:305-312.

16. Andrews G, Creamer M, Crino R, et al. The treatment of Anxiety Disorders: Clinician Guides and Patient Manuals. 2nd ed. Cambridge, UK: Cambridge University Press; 2003.

17. Zinbarg RE, Brown TA, Barlow DH. Hierarchical structure and general factor structure saturation of the Anxiety Sensitivity Index: evidence and implication. Psychol Assess. 1997;9:277-284.

18. Riskind JH, Kleiman EM, Weingarden H, Danvers AF. Cognitive vulnerability to anxiety in the stress generation process: further investigation of the interaction effect between the looming cognitive style and anxiety sensitivity. J Behav Ther Exp Psychiatry. 2013;44: 381-387.

19. Peterson RA, Heilbronner RL. The anxiety sensitivity index. J Anxiety Disord. 1987;1:117-121. 
20. Maruta T, Yamate T, Ito K, Sato M, Iimori M, Kato M. Reliability and validity of the Japanese version of the Anxiety Sensitivity Index. Compr Psychiatry. 2007;48:289-292.

21. Shear MK, Brown TA, Barlow DH, et al. Multicenter collaborative panic disorder severity scale. Am J Psychiatry. 1997;154:1571-1575.

22. Yamamoto I, Nakano Y, Watanabe N, et al. Cross-cultural evaluation of the Panic Disorder Severity Scale in Japan. Depress Anxiety. 2004; 20:17-22.

23. Derogatis LR. SCL-90-R: Administration, Scoring \& Procedures Manual-II, for the R (evised) Version and Other Instruments of the Psychopathology Rating Scale Series. 2nd ed. Towson, MD: Clinical Psychometric Research; 1992.

24. Furukawa TA, Nakanishi M, Sakurai A, et al. Effects of ethyl loflazepate in mood and neurosis-related disorders (ICD-10 JCM): changes in SCL90-R subscale scores. Rinsho Seishinigaku (Clin Psychiatry). 1996;25: 233-240.
25. SPSS. SPSS for Windows (Version 21.0). 2013.

26. Rector NA, Szacun-Shimizu K, Leybman M. Anxiety sensitivity within the anxiety disorders: disorder-specific sensitivities and depression comorbidity. Behav Res Ther. 2007;45:1967-1975.

27. Salkovskis PM, Clark DM, Hackmann A. Treatment of panic attacks using cognitive therapy without exposure or breathing retraining. Behav Res Ther. 1991;29:161-166.

28. Mitchell MA, Capron DW, Raines AM, Schmidt NB. Reduction of cognitive concerns of anxiety sensitivity is uniquely associated with reduction of PTSD and depressive symptoms: a comparison of civilians and veterans. J Psychiatr Res. 2014;48:25-31.

\section{Publish your work in this journal}

Neuropsychiatric Disease and Treatment is an international, peerreviewed journal of clinical therapeutics and pharmacology focusing on concise rapid reporting of clinical or pre-clinical studies on a range of neuropsychiatric and neurological disorders. This journal is indexed on PubMed Central, the 'PsycINFO' database and CAS, and is the official journal of The International Neuropsychiatric Association (INA). The manuscript management system is completely online and includes a very quick and fair peer-review system, which is all easy to use. Visit http://www.dovepress.com/testimonials.php to read real quotes from published authors. 